\title{
Alcohol and burden of disease: Countering myths for effective prevention
}

\author{
Vajira Dharmawardene
}

Consuming alcohol is more or less risky. This needs no reiteration.

However there has always been debate on quantifying this risk and the health impact of alcohol use. This was specially in the background of continuous argument for possible health benefits from low alcohol consumption. ${ }^{1}$

Drugs like alcohol cause harm in multiple ways, both in users and third parties and there are different methods to measure this. A useful way to measure the harm of drugs is to combine the harm to the user and to the others, who are often victims. An example would be an alcohol user who is having liver damage (harm to the user), driving a motor vehicle under the influence of alcohol, injuring a pedestrian (harm to others).

An analysis that combined the harm to users and others using multiple criteria rated alcohol as the most harmful drug (overall harm score 72 out of 100), with heroin (55) and crack cocaine (54) in second and third places. $^{2}$

Harm could be measured in economic terms as well. Based on prevalence-based gross costing approach for Sri Lanka in 2015, the economic cost of the alcoholrelated conditions was USD 885.86 million or $1.07 \%$ of the GDP. ${ }^{3}$ This cost share of GDP by alcohol harm needs to be compared with the Total Health Expenditure in the same year for the country which was at $2.97 \%$ of GDP. ${ }^{4}$

This year, in the most comprehensive attempt to measure the health impact of alcohol use to date, Alcohol Collaborators in Global Burden of Disease 2016 study group came out with finding $5^{5}$ that could have been unexpected for some, but not surprising for others ${ }^{6}$, who knew this was coming. The latter group has long argued that the apparent health benefits of alcohol are due to methodological errors in the studies supporting evidence. ${ }^{7}$

The findings from the 2016 Global Burden of Diseases, Injuries, and Risk Factors Study (GBD) data available for 195 countries and territories, show alcohol use as the leading risk factor for burden of disease as mea- sured by Disability Adjusted Life Years (DALYs), a composite measure of mortality and morbidity, among the 15-49 year age group globally. In this age group, which could be described as the most productive segment of the society, $3.8 \%$ (3.2-4.3) of female deaths and $12.2 \%$ (10.8-13.6) of male deaths could be attributed to alcohol use. Considering all the age groups, alcohol was the 7 th leading cause for burden of disease and accounted for nearly 3 million deaths that year globally. ${ }^{5}$

However the most pertinent amongst the findings of the above study for the long standing debate is the evidence that the level of consumption that minimizes health loss is zero.

The conclusion is based on weighted relative risk curves for each health outcome associated with alcohol use. For example the risks estimated for low level of consumption is considerable with one drink a day (defined as $10 \mathrm{~g}$ of pure alcohol) increasing the risk of developing an alcohol-related disease including cancer, diabetes and tuberculosis by 0.5 per cent. This rises to 7 percent for two drinks and 37 percent for five drinks a day.$\underline{5}$

The implications of these revelations are multiple. Primarily it calls for alcohol control policies aimed at reducing population-level consumption. Secondly, it calls to stop advocating possible health benefits of low level of alcohol consumption. The only way to avoid the health risks associated with alcohol is giving up drinking entirely. Concepts like low risk drinking or healthy drinking are evidently oxymoron.

Debunking myth of healthy alcohol use will not be complete unless effective, evidence based prevention strategies are implemented fully to prevent massive harm associated with alcohol use. Consider the burden from transport related injuries. In 2015 (road) injuries had accounted for $25.5 \%$ of the harm due to alcohol in Sri Lanka. ${ }^{3}$ A paper published in this issue of JRCS gives an example from a semi urban North Central province in Sri Lanka showing approximately $20 \%$ road traffic injuries were related to alcohol use. ${ }^{8}$ This figure could be an underestimate as it was by self report. In 2017, the Sri Lanka police has detected over 70, 000 incidents of 
driving under the influence of alcohol but estimated the real rate could be 3 times the number. ${ }^{9}$

The huge costs involved should drive a rational health and economic policy towards applying evidence based alcohol prevention policies. ${ }^{10}$ The interventions need to address whole populations. The idea that alcohol problems are created mostly by a small group of alcohol dependent individuals who require medical or mental health treatment is false.

An example of application of ineffective strategies is opting for educational interventions to counter the problem of drunken drivers. Only a proportion of them are dependent drinkers. The intervention with most robust evidence for effectiveness to curb alcohol related road traffic injuries and deaths is for random breath testing at checkpoints by the Law Enforcement Officers. ${ }^{10}$ The idea of imposing a legal limit for driving to address the issue has no scientific basis as the alcohol level that is compatible with safe driving, specially for young drivers, is zero. ${ }^{11}$ Alcohol education programmes targeting children in schools are also known to be ineffective. ${ }^{10}$ In an environment in which alcohol is readily accessible and many competing messages are received by children in the form of marketing and social norms that support drinking, mere education does not lead to a sustained behavior change.

Effective evidence based interventions to counter alcohol problems are well known. Making alcohol more expensive and less available and banning alcohol advertising are highly cost-effective strategies to reduce harm from alcohol. Legislative measures to reduce drink-driving and individually directed treatment interventions to at-risk drinkers are also effective. ${ }^{10}$ Latter should not come at the expense of more effective population based action.

Burden of disease due to alcohol is facilitated by unhealthy environments and persistence with mythologies that disregard the tragic human consequences. Alcohol prevention action needs to be driven by evidence for efficacy and not by fancies.

\section{References :}

1. Agarwal DP. Cardioprotective effects of light-moderate consumption of alcohol: a review of putative mechanisms, Alcohol and Alcoholism, Volume 37, Issue 5, 1 September 2002, Pages 409-415.
2. Nutt DJ, King LA, Phillips LD; Independent Scientific Committee on Drugs. Drug harms in the UK: a multicriteria decision analysis. Lancet. 2010 Nov 6; 376(9752):1558-65.

3. Ranaweera $\mathrm{S}$, Amarasinghe $\mathrm{H}$,ChandraratneN et al. (2018)Economic costs of alcohol use in SriLanka. PLoSONE13(6): e0198640.https://doi. org/10.1371/journal.pone.0198640

4. World Health Organization (2017) Health Financing Profile: Sri Lanka. http://apps.who.int/iris/ bitstream/handle/10665/259644/HFP-SRL.pdf?sequence $=1$ \&isAllowed $=\mathrm{y}$ ( accessed on $30 / 10 / 2018$ )

5. GBD 2016 Alcohol Collaborators. Alcohol use and burden for 195 countries and territories, 19902016: a systematic analysis for the Global Burden of Disease Study 2016. Lancet 2018; Sep 22; 392(10152):1015-1035.

6. Chikritzhs T1, Fillmore K, Stockwell T. A healthy dose of scepticism: four good reasons to think again about protective effects of alcohol on coronary heart disease. Drug Alcohol Rev. 2009 Jul;28(4):441-4.

7. Fekjaer HO. Alcohol-a universal preventive agent? A critical analysis. Addiction. 2013 Dec; 108(12):20517.

8. Weerawardena WAK, Thennegadara TGLHM, Priyantha HGV, Chandrasiri PACGS, Disanayaka WSI. Road traffic accidents in Polonnaruwa District: Analysis of patients admitted to a single surgical unit, Journal of Ruhunu Clinical Society, 2018; 1 , 23: $25-29$.

9.http://www.sundayobserver.lk/2018/10/07/stringent-laws-needed-curb-drunk-driving-preciouslives-nipped-bud

10. Anderson P, Chisholm D, Fuhr DC. Effectiveness and cost-effectiveness of policies and programmes to reduce the harm caused by alcohol. Lancet 2009; 373: 2234-46

11. Zwerling C, Jones MP. Evaluation of the effectiveness of low blood alcohol concentration laws for younger drivers. American Journal of Preventive Medicine, 1999, 16, No.1:76-80. 